\title{
Erratum: The Royal Free Hospital Score: A Calibrated Prognostic Model for Patients With Cirrhosis Admitted to Intensive Care Unit. Comparison With Current Models and CLIF-SOFA Score
}

Eleni Theocharidou, Giulia Pieri, Ali Omar Mohammad, Michelle Cheung, Evangelos Cholongitas, Agarwal Banwari and Andrew K. Burroughs

Am J Gastroenterol 2017; 112:193; doi:10.1038/ajg.2016.562; published online 13 December 2016

Correction to: Am J Gastroenterol 2014; 109:554-562; doi:10.1038/ajg.2013.466; published online 4 February 2014

A second affiliation for Ali Omar Mohammad should be added to the existing affiliation:

${ }^{3}$ Department of Thoracic Medicine, Minia University, Elminia, Egypt

\section{Corrigendum: No Change in the Mucosal Gut Microbiome is Associated with Celiac Disease-Specific Microbiome Alteration in Adult Patients}

Valeria D’Argenio, Giorgio Casaburi, Vincenza Precone, Chiara Pagliuca, Roberta Colicchio, Daniela Sarnataro, Valentina Discepolo, Sangman M. Kim, Ilaria Russo, Giovanna Del Vecchio Blanco, David S. Horner, Matteo Chiara, Graziano Pesole, Paola Salvatore, Giovanni Monteleone, Carolina Ciacci, Gregory J. Caporaso, Bana Jabrì, Francesco Salvatore and Lucia Sacchetti

Am J Gastroenterol 2017; 112:193; doi:10.1038/ajg.2016.561; published online 13 December 2016

Correction to: Am J Gastroenterol 2016; 111:1659-1661; doi:10.1038/ajg.2016.227

The title should read:

No Change in the Mucosal Gut Mycobioma Is Associated with Celiac Disease-Specific Microbiome Alteration in Adult Patients 